\title{
GENİŞ BANTLI ÇİFT KUTUPLU ANTEN
}

\author{
Mehmet ÇIYDEM \\ ENGITTEK Mühendislik Ltd, 06460 Balgat, Ankara \\ mehmet.ciydem@engitek.com.tr
}

(Geliş/Received: 05.02.2014; Kabul/Accepted: 04.10.2014)

ÖZET

Bu makalede 1710-2170 Mhz bandında çalışan, geniş bantlı, çift kutuplu askıda yığın plaka anten tasarımı ve geliştirmesi ele alınmıştır. Anten, klasik yama antenlerin beslemesinden farklı olarak, kapasitif kuplaj beslemeli iki adet askıda yığın plaka anten elemanından oluşmaktadır. Kutuplanma çeşitlemesi sağlamak için anten, $\pm 45^{\circ}$ doğrusal kutuplu bağımsız çift kanallı tasarlanmıştır. Anten elemanları, kanallar arasında yüksek izolasyonu sağlamak için yatayda ve dikeyde optimal aralıklarla yerleştirilmiştir. Eleman tasarımdan başlayarak, antenin tasarımı, benzetimleri, üretimi ve ölçümleri yapılmıştır. Geri dönüş kayıpları, kanal izolasyonu, 1şıma örgüsü, kazanç vb anten performansına ilişkin sonuçlar verilmiş ve benzetimler ile ölçümlerin tutarlı olduğu görülmüştür.

Anahtar kelimeler: geniş bant, çift kutuplu, anten, s-parametreleri, 1şıma örgüsü.

\section{WIDEBAND DUAL POLARIZED ANTENNA}

\begin{abstract}
This paper introduces wideband dual polarized stacked suspended plate antenna operating in 1710-2170 Mhz. Antenna consists of two pairs of stacked suspended plate antennas, which have novel capacitively coupled feeding probes different than excitation of classical patch antennas. Antenna is designed to provide independent $\pm 45^{\circ}$ linearly polarized dual channels for polarization diversity. Antenna elements of these $\pm 45^{\circ}$ channels are separated by a distance in azimuth and interleaved by a distance in elevation to have a good isolation between ports. Starting from single element, antenna is designed, simulated, manufactured and measured. Simulations are in good agreement with the measurement in terms of antenna performance parameters; return losses, channel isolation, radiation patterns and gain.
\end{abstract}

Keywords: wideband, dual polarization, antenna, s-parameters, radiation pattern.

\section{GİRIŞ (INTRODUCTION)}

Mobil haberleșme sistemlerinde günümüzde en yaygın kullanılan frekans $(f)$ bölgesi 1710-2170 Mhz'dir. $\mathrm{Bu}$ frekans bandında geniş bantlı çift kutuplu anten tasarımları üzerine literatürde pek çok çalışma (dipole, log-periyodik, askıda yığın anten, yama anten yapıları vb) vardır [1-12]. Genişbant özelliği açısından, log-periyodik [13] ve askıda yığın anten yapıları [14] öne çıkmaktadır. Yüksek izolasyonlu çift kutupluluk özelliği açısından ise dipole [14] ve askıda yığın anten yapıları [15] avantajlıdır. Yama anten yapılarını geniş bantlı yapmak için askıya almak ve uygun şekilde beslemek gerekmektedir (yarık kuplajlı, besleme ucu (probe), kapasitif kuplajlı tersinir L/T/V, veya hibrit uçlar) [39,12]. Askıda yüksekliği arttırmak geniş bantlı olma kabiliyetini geliştirmekle beraber, özellikle yarıkkuplajlı beslemelerde kuplaj ve empedans uyumu zorlaştırmaktadır. Bununla beraber yarık-kuplajlı beslemelerde kanallar arasında yüksek izolasyon sağlamak kolaylaşmaktadır. Geniş bant için askı yüksekliği arttırılırken etkin bir enerji kuplajı ve empedans uyumu, kapasitif kuplajlı tersinir L/T/V şekilli beslemelerle sağlanabilir. Bu bağlamda sadece askıda yığın plaka antenin değil, beslemenin de geniş bantlı tasarlanması gerekmektedir. Ancak bu durumda da kanallar arasında yüksek izolasyon sağlanması zorlaşmaktadır. $\mathrm{Bu}$ açılardan bakıldığından mobil haberleşme için teknik gereksinimleri sağlayan geniş 
bantlı çift kutuplu anten tasarımı önemli bir problemdir.

Merkez frekansı (1940 Mhz) ve çalışma bandı (460 Mhz) dikkate alınırsa, yaklaşık 24\% bant genişliği sağlanmalıdır. Literatürdeki yayınlarda [1-9] sözkonusu bant genişliği için antenin $\mathrm{S}_{11}, \mathrm{~S}_{22} \leq-10 \mathrm{~dB}$ olduğu empedans uyum frekans aralığ1 esas alınmaktadır, yeterli görülmektedir. Fakat pratikte baz istasyonlarında kullanılan profesyonel ürünler [1315], performans gereksinimi olarak $\mathrm{S}_{11}, \mathrm{~S}_{22} \leq-15 \mathrm{~dB}$ seviyesine göre yapılmaktadır. 24\%'lük geniş bandın $\mathrm{S}_{11}, \mathrm{~S}_{22} \leq-15 \mathrm{~dB}$ 'ye göre sağlanması da tasarımı zorlaştırmaktadır. Diğer yandan sinyal-gürültü oranında (SNR) 3 dB daha kazanç sağlamak için kutuplanma çeşitlemesi de (polarization diversity) kullanılmaktadır ve antenler çift kutuplu, en yaygın haliyle $\pm 45^{\circ}$ doğrusal kutuplu olarak tasarlanmaktadır [16]. Fiziksel alan ve boyut sınırlamaları içinde kalarak, anten yapısında çift kutuplu bağımsız kanalları ve bu kanallar arasındaki izolasyon $\mathrm{S}_{21}, \mathrm{~S}_{12} \leq-25 \mathrm{~dB}$ 'i sağlamak ta ayrı bir gereksinimdir $[1,7,8,16,17]$.

Bu çalışmada 1710-2170 Mhz mobil haberleşme (GSM1800, UMTS) baz istasyonları için geniş bantlı, çift kutuplu ( $\pm 45^{\circ}$ doğrusal $)$ anten tasarımı sunulmaktadır. Anten tasarımı, benzetimi, üretimi ve ölçümleri yapılarak sonuçlar (empedans uyumu, kanal izolasyonu, ışıma örgüsü, kazanç) karşılaştırılmıştır. Tasarımın ekonomik, hafif ve üretilebilir olmasına dikkat edilmiştir.

\section{ELEMAN TASARIMI (ELEMENT DESIGN)}

Anten eleman tasarımı bir tarafi toprak olarak kullanılan çift yüzlü FR-4 üzerinde yapılmıştır. FR-4 taban malzemesinin temel özellikleri şöyledir; kalınlık $1.6 \mathrm{~mm}$, bakır kaplama $0.03 \mathrm{~mm}$, dielektrik sabiti $\left(\varepsilon_{r}\right) 4.4$ ve tanjant kaybı 0.02. Anten elemanı, 1şıma yapan askıda plakalar ve kapasitif kuplajlı hibrit tersinir $\mathrm{L} / \mathrm{T} / \mathrm{V}$ şekilli besleme içermektedir. Askıda plakalar ve FR-4 tabaka arası hava ortamıdır $\left(\varepsilon_{0}\right)$. Bu çalışmada; yazar ve diğerlerinin önceki çalışmasındaki [18] askıda antenler ve kapasitif kuplajlı besleme yapıları değiștirilerek ve antenin boyutları ciddi oranda küçültülerek iyileştirmeler yapılmıştır. Bütün parçaların boyutları, yükseklikleri, birbirlerine göre pozisyonları, toprak düzlemin büyüklüğü anten elemanın geri dönüş kaybı, empedans uyumu, 1şıma örgüsü, kazanç gibi tüm tasarım parametrelerini etkilemektedir. $\mathrm{Bu}$ nedenle HFSSv10 ile anten elemanın (Şekil-1) benzetimleri tüm parametreler dikkate alınarak 1710-2170 Mhz için optimize edilmiştir ve Çizelge-1'deki nihai tasarım değerleri elde edilmiştir. $\mathrm{Bu}$ benzetimler esnasında; beslemedeki $h_{3}$ yüksekliğinin 1şıma örgüsü açısından kritik olduğu gözlemlenmiştir. Bu yükseklik arttıkça, besleme ayağı da 1şıma yaparak toplam ışıma örgüsünde asimetri ve istenmeyen 1șımalar yaratmaktadır. Geniş bant özelliği $\left(\mathrm{S}_{11}, \mathrm{~S}_{22} \leq-15 \mathrm{~dB}\right)$ askıda tek plaka ile sağlanamadığı için ikinci bir plakaya (parazitik plaka) daha ihtiyaç duyulmuştur. $\mathrm{Bu}$ ikinci plaka sayesinde hem empedans uyum band1 genişletilmiş hem de antenin kazancı arttırılmıștır. Besleme ayağının V-şekilli olması beslemenin geniş bantlı olmasını sağlamıştır. RF enerjinin askıda plakalara kapasitif kuplajını sağlayan şapka tarzı plakanın büyüklüğü de tasarım ve benzetimlerde önemli olmuştur. Literatürde [4,5,7,12] olan ince ve küçük alanlı şapka plakaların enerji kuplajı ve geniş bantlı olma özellikleri, bu tasarımda önerilen yap1 kadar iyi değildir. Aksi takdirde tasarım hedeflerinin sağlanması mümkün olmamıștır.

Çizelge-1. Anten elemanının boyutları (dimensions of antenna element)

\begin{tabular}{|c|c|c|c|}
\hline Parametre & $\mathbf{m m}$ & Plakalar & mm \\
\hline $\mathrm{h}_{1}$ & 10 & Askida plaka-1 & $60 \times 60$ \\
\hline $\mathrm{h}_{2}$ & 15 & Askida plaka-2 & $50 \times 50$ \\
\hline $\mathrm{h}_{3}$ & 10 & Kapasitif kuplaj besleme plakas1 & $20 \times 20$ \\
\hline
\end{tabular}

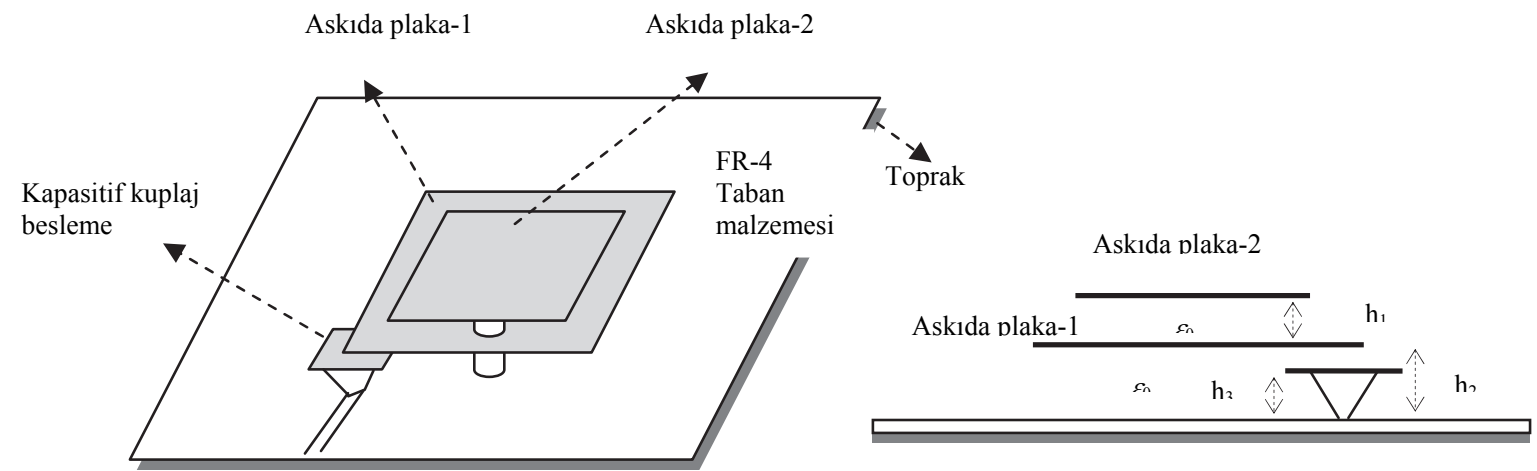

Şekil-1. Anten elemanının yerleşimi ve çizimi (Layout and drawings of antenna element) 


\section{ANTEN TASARIMI (Antenna Design)}

Tek kutuplu tekil anten eleman1, yukardaki gibi tasarlandıktan sonra, geniş bantlı ve çift kutuplu anteni oluşturmak için iki adet tekil anten elemanı kullanılmıştır (Şekil-2). Elemanlardan her biri, birbirine dik $\pm 45^{\circ}$ doğrusal kutuplu bağımsız kanala karşılık gelmektedir. Antenin kanallar arası izolasyonunun $\left(\mathrm{S}_{21}, \quad \mathrm{~S}_{12} \leq-25 \mathrm{~dB}\right)$ ve anten boyutlarının pratikte kullanılabilecek minimum boyutlarda olması gereklidir [17]. Bu maksatla, tekil elemanlar yatayda ve dikeyde aralıklı yerleştirilmiştir. $\mathrm{Bu}$ haliyle tasarım, [18]'den bütün parametreleriyle ciddi miktarda farklılaşmış ve boyutlar $40 \mathrm{~cm} \mathrm{X} 13$ cm'den $17 \mathrm{~cm}$ X 17 cm'e düşürülmüştür. Sözkonusu antenin performans tasarım hedefleri Çizelge-2'de verilmiştir.

\section{BENZETIMLER VE ÖLÇÜM SONUÇLARI (SIMULATIONS AND MEASUREMENT RESULTS)}

HFSSv10 ile benzetimleri ve optimizasyonu yapılan anten tasarımı, üretilerek ölçümleri yapılmış ve sonuçlar karşılaştırılmıştır. Antenin empedans uyumu, geri dönüş kayıpları $\left(\mathrm{S}_{11}, \mathrm{~S}_{22}\right)$ ve kanallar arası izolasyonu $\left(\mathrm{S}_{21}, \mathrm{~S}_{12}\right)$ HP8720D vektör network analizör ile, ışıma örgüsü ve kazancı da yankısız odada ölçülmüştür. Benzetimlerin ölçümlerle uyumlu ve tutarlı olduğu görülmüştür.

1710-2170 Mhz band1 boyunca $S_{11}$ ve $S_{22}$ 'nin -15 dB'den daha düşük olduğu (Şekil-3) ve Çizelge2'deki tasarım hedefinin sağlandığı görülmektedir. $\mathrm{S}_{11}$ ve $S_{22}$ ölçümlerindeki farklar, antenin montajı ve üretiminde olabilecek milimetrik insani hatalardan kaynaklanmaktadır. $\pm 45^{\circ}$ doğrusal kutuplu kanallar arası izolasyona ait $\mathrm{S}_{12}, \mathrm{~S}_{21}$ için benzetim ve ölçüm sonuçları Şekil-4'de verilmiştir ve Çizelge-2'deki tasarım hedefinin sağlandığı görülmektedir. Şekil5 'de ise antenin tüm bant boyunca kazancı verilmiştir. Yarım güç hüzme genişliğinden de tahmin edilebileceği gibi, antenin kazancı frekansa göre bir artış göstermektektedir. Bunun nedeni askıda plakaların boyutlarının frekans arttıkça dalga boyuna göre göreceli büyük kalıp, daha dar 1şın demeti vermesidir. Zira bandın başında (1710 Mhz) dalgaboyu $(\lambda) 17,5 \mathrm{~cm}$ iken bandın sonunda (2170 Mhz) dalgaboyu 13,8 cm'ye düşerken bununla beraber askıda plakaların boyutları $(60 \mathrm{~mm}$ ve 50 $\mathrm{mm})$ sabittir. Sonuç olarak, ölçümlerin ve benzetimlerin birbirleriyle uyumlu ve tutarlı olduğu ve Çizelge-2'deki tasarım hedeflerinin sağlandığ 1 görülmektedir. Antenin 1şıma örgüsü de yankısız odada, bandın ortasında (1940 Mhz) $+45^{\circ}$ kutuplu kanal için yatay ve dikey düzlemde ölçülmüştür (Şekil-6). Bandın ortasında yatayda ve dikeyde $65^{\circ} \pm 2^{\circ}$ aralığında hüzme genişlikleri ölçülmüştür. Kazanç grafiği dikkate alındığında, tüm bant boyunca antenin $65^{\circ} \pm 5^{\circ}$ aralığında hüzme genişliği sonucu çıkarılabilir. Bandın alt bölgesinde $70^{\circ}$ ye doğru, bandın üst bölgesinde de $60^{\circ}$ 'ye doğru yaklaşacaktır. $-45^{\circ}$ kanal için ise, $\pm 2^{\circ}$ derecelik sapmalar içinde, benzer 1şıma örgüleri ve hüzme genişlikleri elde edilmiştir. $\mathrm{Bu}$ hüzme genişlikleri ve 1şıma örgüleri, baz istasyonlarında tipik sektörel uygulamalar için uygundur.
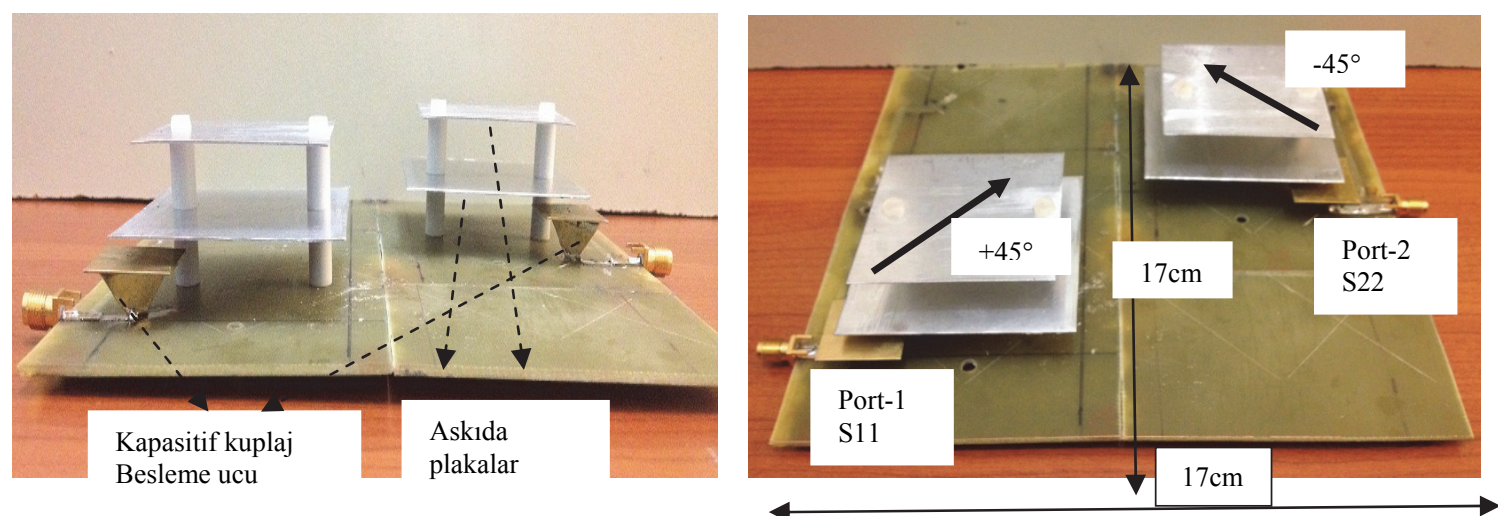

Şekil-2. Antenin ön ve tepe görünüşleri (front and top views of antenna)

Çizelge-2. Anten tasarım hedefleri (Antenna design target parameters)

\begin{tabular}{|c|c|}
\hline Frekans Bandı (MHz) & $1710-2170$ \\
\hline Kazanç (dBi) & $8.5 \pm 1$ \\
\hline Kutuplanma & \pm 45 doğrusal \\
\hline Yatayda Yarım Güç Hüzme Genişliği (HPBW) (derece) & $65^{\circ} \pm 5^{\circ}$ \\
\hline Dikeyde Yarım Güç Hüzme Genişliği (HPBW) (derece) & $65^{\circ} \pm 5^{\circ}$ \\
\hline Kanal izolasyonu (dB) (S12, S21) & $\leq-25$ \\
\hline Empedans uyumu, geniş dönüş kayıpları (dB) (S11, S22) & $\leq-15$ \\
\hline Empedans $(\Omega)$ & 50 \\
\hline
\end{tabular}



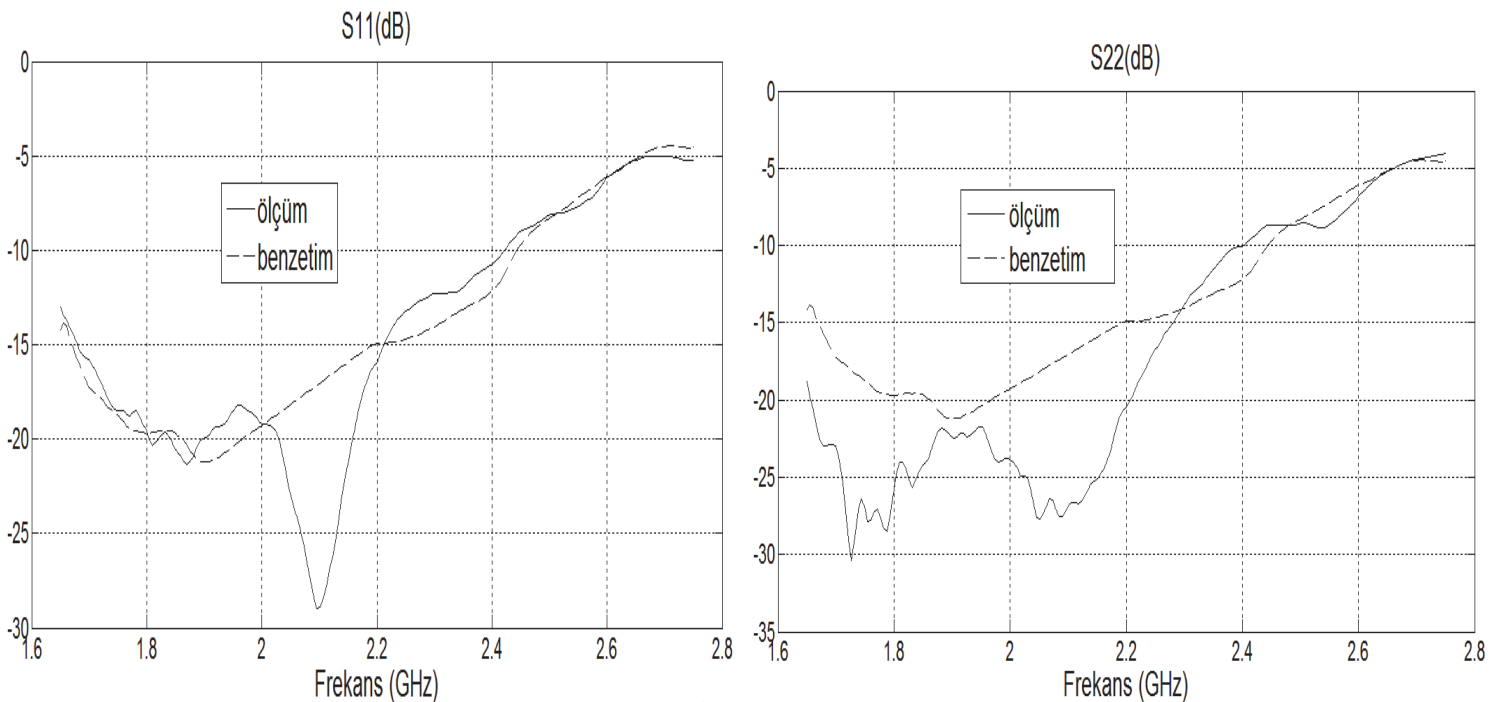

Şekil-3. Kanalların geri dönüş kayıpları (return losses of channels)
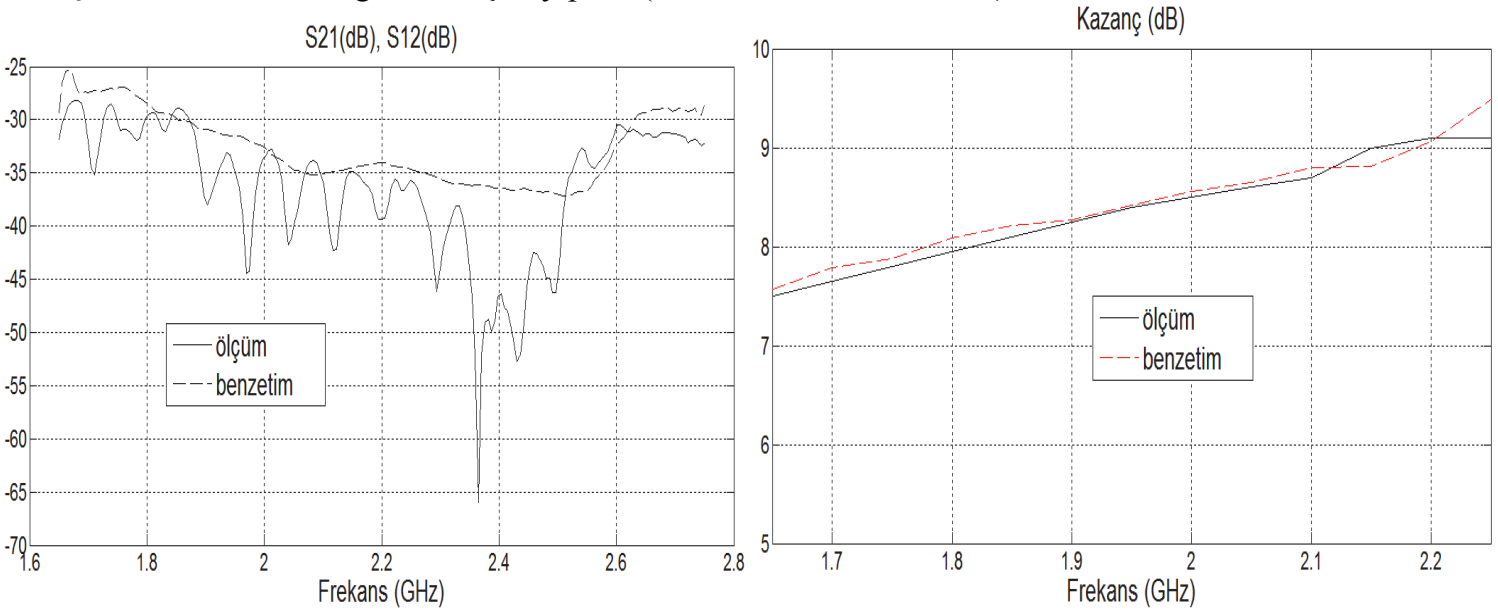

Şekil-4. Kanallararası izolasyon (channel isolation) Şekil-5. Anten kazancı (antenna gain)
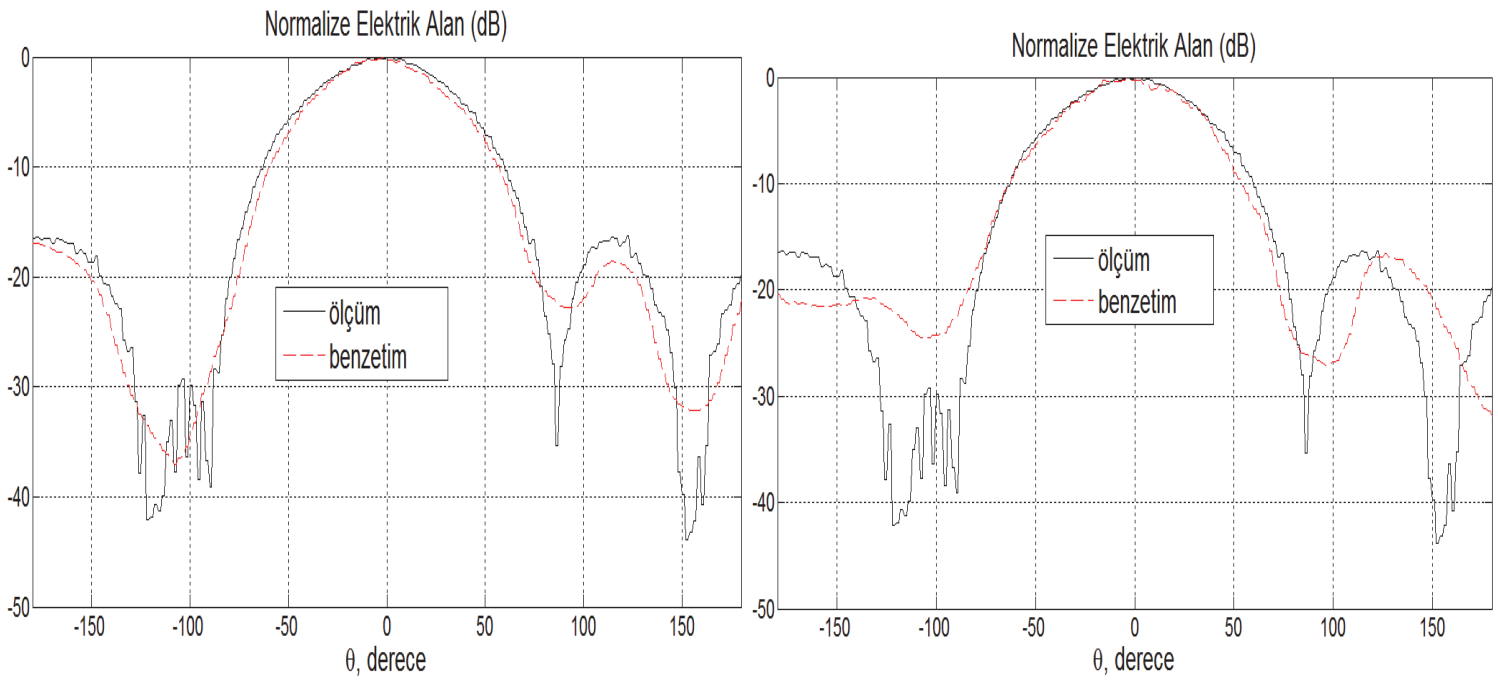

Şekil-6. Antenin yatay ve dikey ışıma örgüsü (Horizontal and elevation pattern of antenna) 


\section{SONUÇ VE TARTIŞMA (CONCLUSION AND DISCUSSIONS}

$\mathrm{Bu}$ çalışmada özel bir besleme yapısına sahip geniş bantlı çift kutuplu anten tasarımı ve geliştirmesi sunulmuştur. Sözkonusu anten 1710-2170 Mhz frekans aralığında çalışmakta olup GSM1800 ve UMTS baz istasyonlarında temel anten yapısı olarak kullanılmaya uygundur. Antenin benzetimi, prototip üretimi ve ölçümleri yapılmıştır. Sonuçlar karşılaştırıldığında, benzetimler ve ölçümlerin uyumlu olduğu ve tasarım hedefi performans parametrelerinin sağlandığı görülmüştür. İleriki çalışmalarda antenin eşdeğer devre modelinin detaylı analizi, değerlerin tespiti ve geniş bantlı çift kutuplu olma özelliğinin, iki ayrı anten elemanı kullanarak değil, tekil bir anten yapısında geliştirilmesi ele alınacaktır.

\section{TEŞEKKÜR (ACKNOWLEDGEMENT)}

Laboratuar ve ölçüm çalışmalarında sağladığ 1 destek ve yardımlardan dolayı hocam Sn. Prof. Sencer Koç'a ve ODTÜ-EE bölümüne çok teşekkür ederim.

\section{SEMBOLLER VE KISALTMALAR (SYMBOLS AND ABBREVIATIONS)}

$\varepsilon_{0}$ : Boş uzay dielektrik geçirgenliği, $(1 / 36 \pi) \times 10^{-9}$

$(\mathrm{F} / \mathrm{m})$

$\varepsilon_{\mathrm{r}}$ : Dielektrik sabiti

$f:$ Frekans $(\mathrm{Hz})$

$\lambda$ : Dalgaboyu (m)

dB: Desibel

GSM: Global System for Mobile communication UMTS: Universal Mobile Telecommunication System HFSS: High Frequency Structure Simulator HPBW: Yarım-Güç Hüzme Genişliği (Half Power Beam Width)

RF: Radyo Frekans

SNR: Sinyal-Gürültü Oranı (Signal to Noise Ratio)

\section{KAYNAKLAR (REFERENCES)}

1. Luo, K., Ding, W. ve et al, "Design of dual feed dual polarization microstrip antenna with high isolation and low cross polarization", Progress in Electromagnetics Research Cilt 36, 31-40, 2013.

2. Serra, A.A., Nepa, P., Manara, G., Tribellini G., ve Cioci S., "A wide-band dual-polarized stacked antenna", IEEE Antennas and Wireless Propag. Lett., Cilt 6, 141-143, 2007.

3. Eldek, A.A., Elsherbeni, A.Z. ve Smith, C.E., "Square slot antenna for dual wideband wireless communication systems", Journal of Electromagnetic Waves and Applications, Cilt 19, No 12, 1571-1581, 2005.
4. Rainee, N.S., "Suspended rectangular/circular patch antennas with electromagnetically coupled inverted microstrip feed with dual polarization/frequency", NASA/CR 2000-210051.

5. Yang, S.S. ve Luk, K-M, "Design of a wide-band L-probe patch antenna for pattern reconfiguration or diversity applications", IEEE Trans. on Antennas \& Propagation, Cilt 54, No 2, 433 438, February 2006.

6. Gao, S. ve Sambell, A., "Dual-polarized broadband microstrip antennas fed by proximity coupling", IEEE Trans. on Antennas \& Propagation, Cilt 53, No 1, 526-530, January 2005.

7. Son, H.-W., "Design of dual-polarised microstrip antenna with high isolation using capacitive feeds", IET Electronics Letters, Cilt 45, No 11, 533-534, May 2009

8. Huang, Y-H., Zhou, S-G., Guo, J-L. ve Chen, YS., "Compact and wideband dual-polarized antenna with high isolation for wireless communication", Progress In Electromagnetics Research, Cilt 38, 171-180, 2013.

9. Xie, J-J., Yin, Y-Z., Ren, J. ve Wang, T., "A wideband dual-polarized patch antenna with electric probe and magnetic loop feeds", Progress In Electromagnetics Research, Cilt 132, 499515, 2012.

10. Wilson et al., "Dual Polarized Based Station Antenna," US Patent No: 6,034,649.

11. Deng et al., "Broadband Dual Polarized Based Station Antenna," US Patent No: 7,629,939.B2.

12. Luk, K.M., Mak, C.L. ve Wong H., "A twin-Lprobe coupled patch antenna with high gain and wide band characteristics", China Patent No: ZL200410042927.8.

13. Andrew Antenna Systems and Solutions, Base Stations Antenna Catalogue, 2013.

14. Powerwave Technologies, Antenna Catalogue, April 2009.

15. Kathrein-Werke KG Rosenheim, 698-6000 MHz Base Station Antennas, Filters, Combiners and Amplifiers for Mobile Communications, Part-I Antennas, December 2012.

16. $\mathrm{Su}, \mathrm{D} ., \mathrm{Fu}, \mathrm{D}$. , Wang, T.N.C. ve Yang H., "Broadband polarization diversity base station antenna for $3 \mathrm{G}$ communication system," Journal of Electromagnetic Waves and Applications, Cilt 22, No. 4, 493-500, 2008.

17. Mak, A.C.K., Rowell, C.R., Murch, R.D., "Isolation Enhancement Between Two Closely Packed Antennas", IEEE Trans. on Antennas \& Propagation, Cilt 56, No:11, 3411-3419, November 2008.

18. İsenlik, T., Bilgiç, M.M., Yeğin, K., Çiydem, M., "GSM/UMTS Dual Polarization Base Station Antenna Design”, IEEE APS/URSI Symp. 2011. 
